\title{
LA PLAZA PÚBLICA COMO ESCENARIO INNOVADOR PARA LA DIVULGACIÓN CIENTÍFICA: PROYECTO VILLA DE LA CIENCIA
}

\author{
Dra. Verbena Córdula Almeida \\ Universidade Estadual de Santa Cruz (UESC), Ilhéus, Brasil \\ vcalmeida@uesc.br \\ Dr. Lisandro Diego Giraldez Alvarez \\ Universidade Estadual do Sudoeste da Bahia (UESB), Vitória da Conquista, Brasil \\ giraldezli@gmail.com
}

Recibido el 24 de octubre de 2018

Aceptado el 26 de diciembre de 2018

\section{Resumen}

A pesar de que 61\% de la población brasileña se interesa por la Ciencia, el Estado brasileño no demuestra acompañar eses intereses si analizamos los cortes presupuestarios que el sector está sufriendo durante los últimos años. Sin embargo, la comunidad académica y científica realiza acciones de divulgación científica con el objetivo de aproximar la ciencia a la sociedad. Así, el Departamento de Ciências e Tecnologías (DCT), de la Universidade Estadual do Sudoeste de Bahia (UESB) ha desarrollado el proyecto de extensión universitaria denominado Vila da Ciência (Villa de la Ciencia en español), el cual, además de colaborar con proyectos en Química, Biología, Matemáticas y Física, ofrece ciclos de conferencias que son presentados en diferentes escuelas, principalmente públicas, y privadas. Son charlas presentadas por estudiantes y profesores de los cursos de postgrado de la UESB sobre sus investigaciones y utilizando un lenguaje adecuado a los jóvenes de las instituciones que lo solicitan. El foco principal está orientado hacia las escuelas públicas debido a que en ellas es donde los responsables del proyecto notaron el mayor índice de rechazo al estudio de las Ciencias. Con la finalidad de democratizar el acceso al conocimiento científico y tecnológico, así como aproximar la Universidad a la sociedad, una de las iniciativas más innovadoras del proyecto es la realización del evento denominado Vila da Ciência el cual consiste en una exposición anual de los trabajos de los estudiantes en la principal plaza pública de la ciudad de Jequié, Estado de Bahia, Brasil.

Palabras clave: Plaza Pública, Divulgación Científica, Sociedad, Brasil. 
THE PUBLIC SQUARE AS AN INNOVATIVE PLACE FOR SCIENTIFIC DIVULGATION: VILA DA CIÊNCIA PROJECT

\begin{abstract}
Despite the fact that $61 \%$ of the Brazilian population is interested in Science, the Brazilian State does not show any interest in these facts if we analyze the budget cuts that the sector is suffering during the last years. However, the academic and scientific community carries out scientific dissemination actions with the aim of bringing science closer to society. Thus, the Departamento de Ciências e Tecnologías (DCT) of the Universidade Estadual do Sudoeste de Bahia (UESB) has developed the university extension project called Vila da Ciência (in Portuguese), which, in addition to collaborating with Projects in Chemistry, Biology, Mathematics and Physics, offers cycles of conferences that are presented in different schools, mainly public, and private. These are talks presented by students and professors of the UESB postgraduate courses about their research and using a language appropriate to the young people of the institutions that request it. The main focus is oriented towards public schools because it is in them where those responsible for the project noticed the highest rate of rejection of the study of Sciences. In order to democratize the access to scientific and technological knowledge, as well as to bring the University closer to society, one of the most innovative initiatives of the project is the realization of the event called Vila da Ciência which consists of an annual exhibition of the works of the students in the main public square of the city of Jequié, State of Bahia, Brazil.
\end{abstract}

Keywords: Public Square, Scientific Divulgation, Society, Brazil.

\title{
Como citar este artículo:
}

Almeida, V. y Giraldez, L. (2019). "La Plaza Pública Como Escenario Innovador Para La Divulgación Científica: Proyecto Vila Da Ciência", en Perspectivas de la Comunicación, Vol. 12, n 1. pp. 117-133. 
Introducción

\section{La ciencia en América Latina}

I papel que tiene la ciencia en nuestra vida es fundamental, no sólo porque nos brinda con los avances tecnológicos, sino también porque nos ayuda como pensar e interpretar el mundo y las relaciones humanas del universo (Giraldez et al, 2013: 28).

En un epígrafe de la obra "¿Es necesaria la ciencia?" (1990), de Max Perutz (1990:15), el primer presidente electo luego de la descolonización de la India, Jawaharlal Nehru, traduce esta necesidad: "¿Quién, en realidad, podría permitirse hoy menospreciar la ciencia? En cada época tenemos que buscar su ayuda. El futuro pertenece a la ciencia y a los que se hacen amigos de ella".

En América Latina, sin embargo, la ciencia normalmente no es una prioridad, sobre todo porque no hay una preocupación sistemática en establecer políticas públicas en ese ámbito, lo que pone a la región en desventaja respecto a los países considerados desarrollados. Cuando nos referimos a política pública lo hacemos pensando en el conjunto de medidas adoptadas por el Estado para intervenir en determinada realidad, en este caso, la científica.

Esas medidas traducen la percepción que tiene el Estado sobre la realidad científica y sobre su concepción de ciencia y del rol que ésta puede desempeñar en el desarrollo de la sociedad. El momento actual es propicio para pensar la ciencia en nuestra región, principalmente si consideramos los cambios de rumbo que están adoptando los gobiernos de la mayoría de los países, que, en muchos casos, significan retrocesos en sectores estratégicos para el desarrollo de los Estados.

Angelo (2017) escribió respecto a la misma cuestión, tratando las marchas realizadas en el año de 2017 por los investigadores en São Paulo en protesta por el déficit de los presupuestos, así como la iniciativa de 23 premios Nobel y de 9 de las sociedades científicas de Brasil que advertían, en septiembre del mismo año, al presidente Temer sobre las incertidumbres en la financiación en curso que amenazan con el desmantelamiento de los grupos de investigación y provocan una fuga de cerebros (Angelo, 2017:166).

Un estudio realizado por el Centro de Gestão em Estudos Estratégicos (CGEE), en conjunto con el Ministério de Ciência, Tecnología e Innovação (MCTI) realizó un sondeo que reveló que el $61 \%$ de los brasileños se interesaban por la ciencia superando, incluso, el interés demostrado por los deportes (56\%). Es importante destacar que, de acuerdo con este trabajo, el interés de los brasileños por la ciencia es más alto que el interés de los estadounidenses. A pesar de ese interés por la ciencia, el $88 \%$ de esas mismas personas entrevistadas no supieron informar el nombre de ninguna institución científica nacional, así como el $94 \%$ de ellas no pudieron citar el nombre de ningún científico brasileño (Brasil, 2015). En comparación con el resultado de una encuesta similar realizada en 2010 por el Ministério de Ciência, Tecnología e Innovação (MCTI) y el Museu da Vida (Casa de Oswaldo Cruz / Fiocruz), el interés por la ciencia sufrió una leve caída (del $65 \%$ en 2010 al $61 \%$ en 2015). La falta de información sigue siendo alta. En 2010, el $71 \%$ de los muy interesados en 
ciencia y tecnología no supieron informar el nombre de ninguna institución científica de Brasil y el $82 \%$ no conocía el nombre de ningún científico brasileño (Castelfranchi et al, 2012:1170).

Aunque haya habido una caída del interés en los cinco años que separan las dos encuestas, los resultados derriban las tesis de que los brasileños no se interesan por esas áreas. Sin embargo, no podemos perder de vista el hecho de que esos trabajos demuestran la fragilidad de las políticas públicas dirigidas a la promoción de la cultura científica en el país, lo cual es muy preocupante dada la importancia de la ciencia para cualquier sociedad.

Petherick afirma que Brasil no es la única nación sudamericana donde los científicos están luchando debido a la terrible situación económica. Conforme subraya, Argentina, donde los científicos tienen la mayoría de los premios Nobel de la región, también enfrenta un futuro incierto y potencialmente sombrío. "Ambos países han sufrido cambios políticos hacia la derecha en los últimos años, al elegir gobiernos que descartan el valor de la financiación de la ciencia" (Petheric, 2017:249). En la Argentina, los presupuestos destinados a las actividades científicas y tecnológicas sufrieron cortes significativos, lo que viene suscitando preocupaciones por parte de su comunidad científica. Por ejemplo, en 2012, hubo un incremento significativo de los presupuestos para las universidades que se pudo verificar, entre otras cosas, a través de la apertura de nuevas instituciones y de la recomposición de los salarios de los docentes y del personal administrativo. El subsistema universitario continuó diversificándose en medio de su expansión con distintas iniciativas de las agencias del sector que dieron cuenta de la búsqueda por una mayor articulación entre las actividades de investigación y desarrollo (Unzué y Rovelli, 2017: 247).

Aún conforme esos mismos autores, entre los años 2003 a 2007 los objetivos principales de las políticas públicas en los contextos científico e tecnológicos de Argentina buscaron fortalecer el conocimiento científico-disciplinar, así como el desarrollo económico, social y productivo. "La fijación de temas prioritarios y la búsqueda de resolución a problemas concretos combinó la preocupación por el desarrollo regional con una distribución más equitativa de los recursos financieros...". (Unzué y Rovelli, 2017: 256).

Sin embargo, ese panorama cambió significativamente. Según Cebrelli y Arancibia (2017) cuando miramos las políticas públicas y comparamos el gobierno anterior y el actual, no hay como no identificar "la discrepancia". Respecto al contexto de la producción de conocimientos, los autores señalan la necesidad de la sociedad argentina definir y decidir qué modelo de ciencia necesita; y llaman la atención para lo que califican de "operación política" existente en Argentina, la cual tiene el objetivo de anular toda clase de debate respecto a políticas públicas. Sostienen, que se trata de poner en práctica una política de falta de financiamiento a la producción científica argentina en su conjunto, con la doble intención de arrasar con la investigación (Cebrelli y Arancibia, 2017:49).

En Chile la situación no es muy distinta. En 2017, el presupuesto del Gobierno Central chileno dirigido para la ciencia, la tecnología y la innovación representaba solamente un $0,36 \%$ del PIB, proporción que se mantuvo relativamente estable en esa década, excepto en los últimos 10 años, cuando la variación significativa se dio entre 2008 y 2009: pasó de un $0,27 \%$ a $0,35 \%$. Se observa que existe una tendencia levemente creciente entre los años 2008 y 2011, y luego una caída hasta estabilizarse en torno al 1,6\% del presupuesto entre los años 2012 y 2017 (Balbontin et al, 2018:17). De acuerdo con los últimos autores citados, hay un consenso sobre "la insuficiente coordinación pública y esfuerzo a nivel de país en el 
esquema vigente, que se explica en parte por la histórica ausencia de una política y estrategia coherente y consistente en esta área". Uno de los muchos aspectos en que esto se ve reflejado es en la consolidación de información presupuestaria que pueda servir como insumo para la discusión pública.

Respecto a la situación de Brasil, la comunidad científica pasó todo el año de 2017 buscando revertir los recortes anunciados para el presupuesto de ese año. La propuesta inicialmente presentada por el gobierno federal, reducía en dos tercios el monto invertido en 2011, como fruto de los cortes anuales que se intensificaron especialmente en 2017. Después de ese gran movimiento, hubo una disminución de los perjuicios, sin embargo, el presupuesto de 2018 se presentó aún menor - alrededor del 19\% con relación al año anterior. El presupuesto del Ministério de Ciência, Tecnología, Innovações e Comunicações (MCTIC) disponible para inversión en becas e investigaciones se redujo según cálculos del propio ministerio $^{1}$, en aproximadamente, de 4,5 para 4 mil millones de reales. El valor exacto de la contingencia es de 477 millones de reales.

\section{Universidad y divulgación científica}

Si los esfuerzos de los Estados son mínimos en el sentido de promover la actividad científica y tecnológica - y en los casos citados, la situación se agrava porque los presupuestos están siendo reducidos - lo mismo se puede afirmar cuando pensamos en la divulgación del conocimiento científico.

Sanabria-Rangel et al. (2015) señalan que, considerando la universidad en cuanto organización que tiene entre sus actividades la generación, difusión y transferencia de saberes, su participación es imprescindible en la nueva economía, la cual pone la construcción de conocimientos como un factor estratégico. La investigación es un proceso indispensable para la producción científica y, por lo tanto, para la ciencia, la tecnología y la innovación. El tema está directamente relacionado con la capacidad que tiene un país de superar desafíos, promover el desarrollo económico y sociocultural, además de mejorar la calidad de vida de los ciudadanos y ciudadanas. Una nación que no esté efectivamente comprometida con la generación de nuevos conocimientos científicos y capacitada para desarrollar aplicaciones tecnológicas identificadas con sus demandas, tiene amenazada su soberanía y está marcada para la subalternidad (Bueno, 2014:3).

Es imprescindible para cualquier país el desarrollo científico y tecnológico, junto con mecanismos de comunicación, para obtener una divulgación científica, mismo sabiendo que se trata de una amplia y delicada discusión (Lima y Viana, 2017). Según esos autores, investigación y desarrollo son algunos de los principios fundamentales para promover la transformación en cualquier país, así como también para fortalecer la economía y la sociedad.

En el prólogo escrito para la obra "La divulgación científica: estructuras y prácticas en las universidades", Perea $(2017)^{2}$ afirma que lograr con que los conocimientos científicos

\footnotetext{
1Recuperado de https://ciencia.estadao.com.br/noticias/geral,orcamento-da-ciencia-e-tecnologia-tem-r-477milhoes-bloqueados,70002179415

2Céullar, M.P.; Martin-Pena, D., Moreno, A. (2017). La divulgación científica: estructuras y prácticas en las universidades. Barcelona: Gedisa. Recuperado de:

https://books.google.com.br/books?h|=pt\&|r=\&id=ENEmDwAAQBAJ\&oi=f $n d \& p g=P T_{3} \& d q=$ divulgacion+cient $\% C_{3} \% A D$ fica\&ots=1syncyEoOV \&sig=m-

fuvpyVDkuLp3boYC 4 kEhAIT8\#v=onepage \&q=divulgacion $\% 20$ ient $\% \mathrm{C}_{3} \% \mathrm{AD}$ fica\&f=false
} 
difundan de modo transversal a los diferentes grupos, democratizar el acceso de las minorías y de las mayorías a eses conocimientos si vuelvo "artículo de primera necesidad. "No se trata únicamente de una comunicación de la ciencia que hable de las investigaciones en proceso o avances de punta...se trata de aquello que le permite a quién no está cercano a la investigación ni a la academia entender..." (Perea, 2017:9).

Esa manera de presentar la necesidad de la universalización del conocimiento científico apuntada por Perea pone de relieve el papel que deben desempeñar las instituciones universitarias, una vez que, como una de las principales productoras de conocimientos, deben buscar mecanismos a través de los cuales esos conocimientos sean diseminados e utilizados por la sociedad. En este sentido, Céullar, Martín-Pena y Moreno subrayan la necesidad de las universidades suplir la carencia de conocimientos sobre ciencia que encontramos en los medios de comunicación. Para esos autores, las universidades tienen la obligación de usar el nuevo contexto digital para llenar ese vacío. Subrayan la imposición del modelo comercial de las empresas mediáticas como factor que corrobora con la poca importancia dada a la divulgación científica por parte de ese segmento y que, por ello, cabe a las universidades encontrar formas para llevar a cabo dicha divulgación.

Pereira y Koshiyama (2017) afirman que cabe aliar al papel de la universidad la promoción de la divulgación científica para el público laico en espacios no formales, como una manera de alinearse a su papel social, y, consecuentemente, provocar el desarrollo pleno de la ciudadanía en el entorno. La ciencia y la tecnología tienen hoy grandes posibilidades de impulsar transformaciones sociales y desarrollos económicos y culturales a medida que contribuyan con los procesos de superación de agudos problemas de pobreza, violencia, injusticia y desigualdad (Sobrinho, 2014:660).

De acuerdo con Unger y Polt (2017), el triángulo del conocimiento, o KT, ha ganado importancia en los últimos años como un marco de políticas de innovación especialmente en la Organización para la Cooperación y el Desarrollo Económico (OCDE) y en la Unión Europea, porque considera un sistema integrado de enfoques que interconectan investigación, educación e innovación. Las inversiones e iniciativas en apoyo a la educación, la ciencia, la tecnología y la innovación son fundamentales para subsidiar políticas de superación del estancamiento de la productividad de los factores de producción nacionales y, además, promover el desarrollo económico y social a largo plazo. Sin un esfuerzo conjunto y cooperativo entre los sectores público y privado, difícilmente Brasil logrará sostener ciclos largos de elevación de la productividad y promover una alteración profunda de la estructura productiva (Turchi y Morais, 2017:8).

El hecho de que América Latina no invierta en la producción de conocimiento sirve como parámetros para pensar, por ejemplo, cuestiones más pragmáticas, que hacen de los países del mundo más o menos innovadores. La encuesta realizada por la Universidad de Cornell, Estados Unidos (2018), que mide el Índice Global de Innovación33, apunta que Brasil ocupa la $64^{a}$ posición en el ranking mundial en cuanto a ese aspecto, lo que significa una escalada de cinco posiciones si consideramos la $69^{a}$ la colocación en la encuesta realizada en 2017 por la misma institución. Aunque Brasil haya avanzado en el último año, ocupa una posición muy inferior en relación a los países que están en la cima de la misma encuesta (Suiza, países bajos, Suecia, Reino Unido).

3 Recuperado de https://www.globalinnovationindex.org/Home. 
Sin embargo, a pesar de la falta de comprometimiento del Estado con la ciencia no impide que la comunidad académica y científica realicen acciones cuyo propósito sea el de aproximar a la sociedad de la ciencia y promover la divulgación de la producción científica realizada en las instituciones de enseñanza superior, ya sea a través de la creación de sitios web de divulgación, la realización de seminarios o, a través de proyectos de innovación en los distintos niveles educativos. Uno de esos casos es un proyecto de extensión, continuo, desarrollado por el Departamento de Ciências e Tecnologías (DCT), de la Universidade Estadual do Sudoeste de Bahia (UESB), denominado como el Vila da Ciência (Villa de la ciencia en español).

\section{El Proyecto Vila da Ciência}

Como vimos, son muchas las visiones que comparten la idea de que tanto la producción como también la divulgación científica son imprescindibles a las sociedades, así como el papel que deben jugar las universidades en los dos ámbitos, sobre todo debido a su función social. El ideal sería la existencia, por parte de todos los Estados, de políticas científicas cuyos objetivos estuvieran centrados tanto en la producción como también en la divulgación y democratización del conocimiento científico, lo que está muy lejos de ocurrir en la región latinoamericana.

En este contexto los esfuerzos individuales de investigadores e instituciones tienden a ser las iniciativas más palpables, las cuales, normalmente, son puestas en práctica con muchas dificultades en virtud de las carencias presupuestarias que se dan en consecuencia de esa ausencia de políticas consistentes que visen la promoción de la cultura científica. Interesante es el proyecto Vila da Ciência, cuya finalidad es la elaboración creativa e innovadora de proyectos en Ciencias y Tecnología (Química, Física, Matemática, Biología), por parte de estudiantes de Educación Infantil, Fundamental, Enseñanza Media, así como también de los estudiantes de los cursos de graduación y post-grado de la propia Universidade Estadual do Sudoeste da Bahia (UESB).

La Universidad Estatadual do Sudoeste de Bahía (UESB) es una institución multicampus, con sede en la tercera ciudad más grande del Estado, la ciudad de Vitória da Conquista ${ }^{4}$, situada en la Mesoregión del Centro-Sur de Bahía 5 , con más de 348 mil habitantes, según datos de 2017 del Instituto Brasileiro de Geografía e Estadística (IBGE).

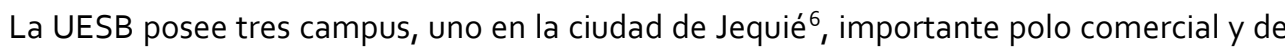
servicios de la región, con una población de 162.209 habitantes y, otro, en la ciudad de Itapetinga7, que se destaca como polo pastoril del Estado, con una población de 77.533 habitantes. Estas subregiones, formadas por un bloque de municipios, presentan dinamismo demográfico relevante, contando con una economía rural relativamente diversificada (café, pecuaria, y algún cacao), además de alguna actividad industrial y significativa oferta de servicios.

4 Tercera ciudad más poblada de Bahía, con 338.885 mil habitantes.

5 Bahía es una de las 27 unidades federativas de Brasil, situada en la Región Nordeste. La capital es Salvador, cuarto municipio más poblado del país (14,8 millones de habitantes). Además de ello, hay otros municipios influyentes en la red urbana bahiana, como las capitales regionales Feria de Santana, Vitória da Conquista, Barreiras, el bipolo Itabuna-llhéus y el bipolo Juazeiro-Petrolina, siendo este último un municipio pernambucano y núcleo, junto con Juazeiro. La mitad de la población de Bahía está concentrada en apenas 35 de las 417 ciudades del estado. Este es el análisis realizado por el Instituto Brasileño de Geografía y Estadística (IBGE) 6 Décima ciudad más poblada de Bahía, con 155.800 mil habitantes. $726^{\text {a }}$ ciudad más poblada de Bahía, con 75.470 mil habitantes. 
La institución tiene 38 años y es actualmente una de las principales responsables de la formación de profesionales en el interior de Bahía y Norte de Minas Gerais, siendo una de las principales referencias de Enseñanza Superior del Norte/Nordeste brasileño. Además de ofrecer docenas de cursos de graduación presencial y semipresencial, también posibilita el ingreso en cursos de postgrado, como especializaciones, maestrías y doctorados.

El proyecto Vila da Ciência es desarrollado, principalmente, en escuelas públicas debido a que en ellas es donde sus idealizadores notaron el mayor índice de rechazo al estudio de las Ciencias, a pesar de que, paradójicamente, de allí es oriundo el mayor número de estudiantes que cursan Química, Sistemas de Información y Biología. De acuerdo a la coordinadora del proyecto, la profesora Alcione Ribeiro, esa contradicción se da en general por cuenta de una elección basada en la baja competencia en el proceso selectivo para la universidad y la nota baja de corte en el Examen Nacional de Ingreso (Enem). "Nuestra intención es hacer que los alumnos se interesen más por Ciencia y que busquen la carrera científica por identificación, no por esa ser la última opción" (Ribeiro, 2018). Aún según ella, a pesar de esa contradicción, el proyecto, que empezó hace 14 años, ya alcanzó varias escuelas privadas también, algunas de ellas ya participan hace algunos años y ya tienen la Vila da Ciência en sus calendarios académicos.

La profesora afirma que la mayoría de los alumnos reciben muy bien el Vila da Ciência, pues lo entienden como un buen apoyo a su trabajo. Pero subraya que existen escuelas y profesores que lo rechazan, por entender el proyecto como más trabajo ante la cantidad que ya tienen. La coordinadora señala que algunas escuelas no entienden bien cuál es la relevancia de la iniciativa y no se interesan. "Pero los discentes suelen gustar mucho de la idea y muchas veces son ellos quienes animan al profesor a participar" (Ribeiro, 2018).

La participación de los becarios en dicho proyecto es fundamental. Según Ribeiro (2018), además de los becarios, los voluntarios son el alma del proyecto, puesto que son ellos que van hasta las escuelas a presentar la idea y se ponen a disposición para ayudar al docente con sus trabajos. Inicialmente los docentes de la UESB que participan del proyecto, además de los becarios y de los voluntarios se dirigen al mayor número de escuelas posible, sin que haya una solicitud. Después esperan el llamado de las escuelas para que los monitores puedan ayudar a los profesores en la realización de los proyectos en las escuelas.

Los registros, que solamente fueron computados desde el año $2012^{8}$ dan cuenta de que el Vila da Ciência ya logró alcanzar 2.500 personas solamente en las escuelas. Además de desarrollar proyectos en Química, Biología, Matemáticas y Física, la Vila da Ciência ofrece ciclos de conferencias para ser presentados en las escuelas de Educación Básica. Son charlas impartidas por estudiantes y profesores de los cursos de postgrado de la UESB, acerca de sus investigaciones, utilizando lenguaje adecuado a los jóvenes de las escuelas que lo solicitan.

Fotografía 1: Palestra del profesor Adílio Macedo (2016)

8 Aunque el proyecto haya iniciado en 2004, la profesora Alcione Ribeiro subraya que asumió la coordinación en 2012, cuando se iniciaron los registros. 


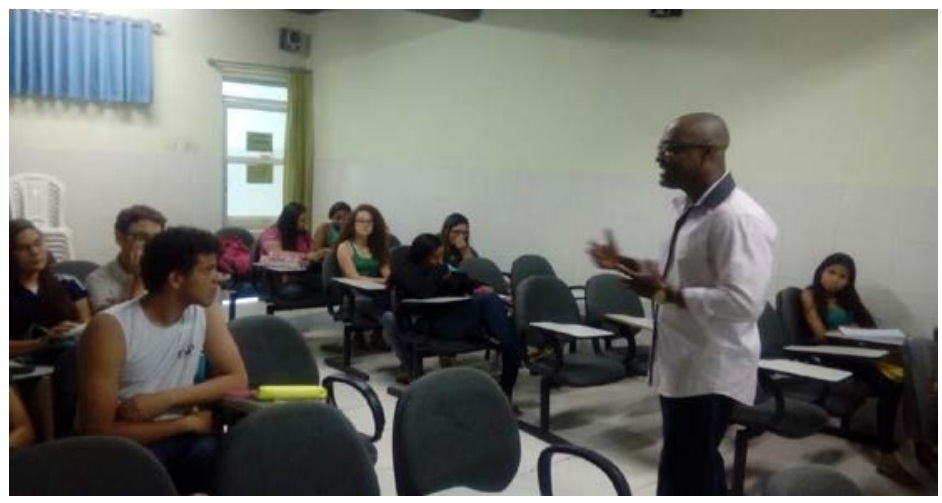

Fuente: Sitio web del Proyecto "Vila da Ciência"

Considerándose que la observación tiene importancia para el desarrollo de procesos que implican la ciencia y la construcción del conocimiento científico (Vogt et al, 2018:72), otra actividad realizada en el Proyecto Vila da Ciência es La Ciencia en Foco, un concurso de fotografía científica, cuyo objetivo es incentivar el arte de la fotografía relacionada con la ciencia y la tecnología, así como la mirada científica sobre las cosas que nos rodean en lo cotidiano. Aún conforme Vogt (2018), se trata de "un lenguaje no verbal que contribuye a la exposición de investigaciones teóricas y aplicadas, en manifestaciones artísticoculturales y como coadyuvante eficaz en innumerables descubrimientos científicotecnológicos". Para esas autoras, en la enseñanza regular de Ciencias, en especial en experimentos, la fotografía puede ser utilizada como medio eficaz de observación y también para el registro de fenómenos. Los profesores de la Vila da Ciência corroboran esa concepción y han trabajado en este sentido. $Y$ muchos frutos salen de los experimentos fotográficos como los ejemplos a seguir.

Fotografía 2: Foto Curvas de Chispas

$1^{\circ}$ lugar (2016), de José Bittencourt Neto

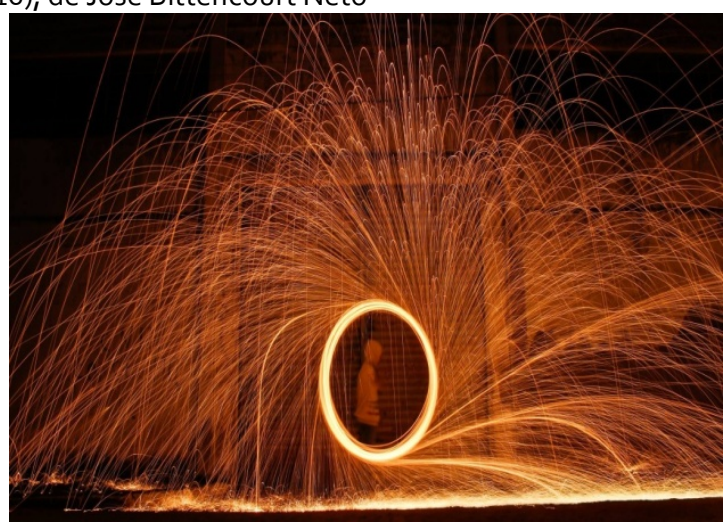

Fotografía 3: Radiación luminosa 
$3^{\circ}$ lugar (2017) de Kandria Cezar

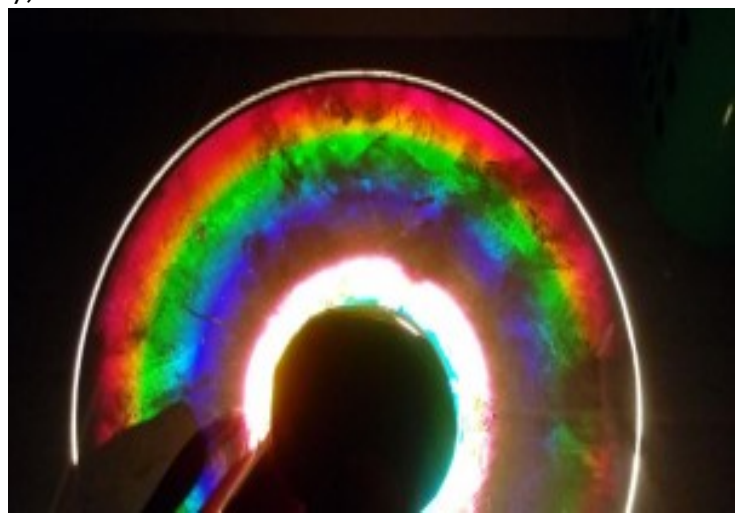

Fuente: Sitio web del Proyecto "Vila da Ciência"

Uno de los eventos de destaque del proyecto es la realización de la exposición anual en la Plaza Ruy Barbosa, en el Centro de la ciudad de Jequié, que permite llevar los trabajos de los estudiantes más allá de los muros de las escuelas y universidades, a fin de alcanzar un público mucho mayor y más variado. En cada edición de este importante evento científico varios talentos han sido descubiertos, abriendo nuevas perspectivas para el futuro de los estudiantes que participan del proyecto. Es precisamente en este ámbito científico-cultural que los integrantes del proyecto convocan a todos los colegios a participar de esta lucha para el enriquecimiento científico de la región. Esa iniciativa corrobora con la visión de una Universidad que se compromete con el medio, pues, como subrayan Castro y Fuentes (2018:621): "lo más importante a observar no es lo institucional, sino el modo en que el conocimiento producido en el territorio... permea, dialoga y construye el conocimiento bajo una epistemología intertranscultural, intertransdisciplinaria".

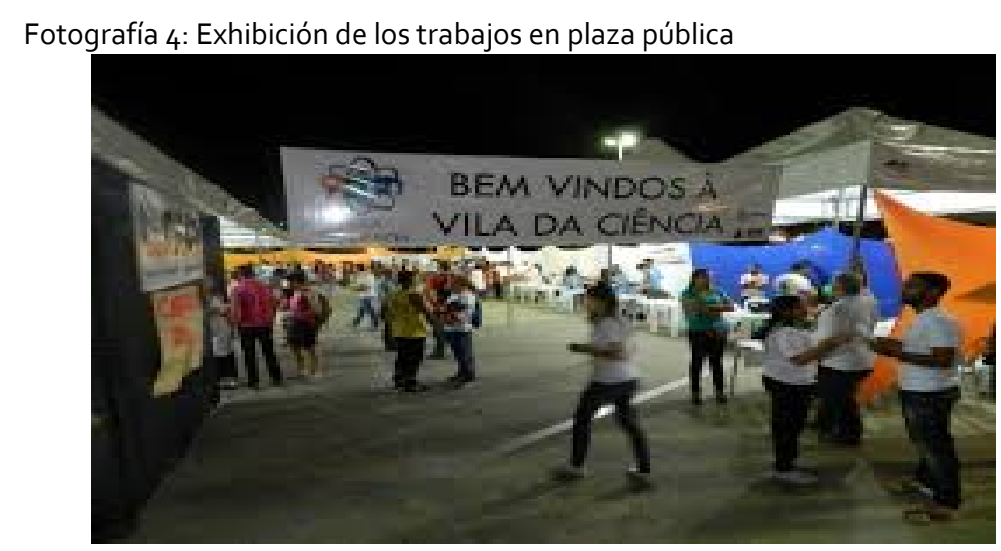

Fuente: Noticias Jequié

Los trabajos presentados en forma de experimentos y pósteres son evaluados por una Comisión Científica y reciben el premio Wilson Rocha ${ }^{9}$, en forma de medallas y trofeo. Se consideran los mismos criterios en la evaluación de los resúmenes, que incluyen interdisciplinaridad; base teórica para explicación del funcionamiento/entendimiento del

9 El idealizador del proyecto. 
trabajo; aplicabilidad real para la ciudad de Jequié y su región, dada sus necesidades específicas; tipos de materiales utilizados (bajo coste reciclados o reaprovechados); tecnologías sostenibles; trabajo realizado individualmente (excluido un probable orientador).

Para cada elemento arriba descrito, se asigna una nota de o a 10. Los tres (3) trabajos con las mayores notas en cada categoría son los premiados. En el caso de que haya más de tres (3) trabajos con nota igual en las primeras colocaciones, se forma un ranking con las mayores notas, siguiendo el orden anterior de los criterios. Persistiendo el empate, la Comisión Científica atribuye el voto de minerva y selecciona a los tres primeros trabajos, obedeciendo a las notas recibidas, en las siguientes categorías: 1. Estudiantes de Graduación y Postgrado; 2. Estudiantes de la Enseñanza Media y Técnica; 3 . Estudiantes de la Enseñanza Fundamental II; 4. Estudiantes de la Enseñanza Fundamental I; 5. Estudiantes de Educación Infantil; 6 . Personas de la comunidad en general que no tengan conexión con una institución de enseñanza formal. Se premia, también, al profesor que obtiene el mayor número de trabajos premiados.

También hay premios para los trabajos que más se destaquen en los 3 días de exposición, conforme elegidos por el público visitante. El trabajo con la mayor puntuación entre todos los presentados en la Vila da Ciência recibe el Premio Innovación, siendo considerado el mejor trabajo de todos y quedando por encima de la clasificación de las otras categorías de premios. Además de los premios por la presentación de trabajos, existe también, la premiación del Concurso de Fotografía Científica Amadora Ciencia en Foco. Por último, la escuela que obtenga el mayor número de méritos científicos (trabajos premiados) recibe el Mérito Científico del año de la Vila da Ciência. En caso de empate, gana la escuela que tenga notas más altas en la evaluación.

Para el año 2017, hubo la concesión de cinco becas de Iniciación Científica Junior, del Conselho Nacional de Pesquisas (CNPq) a aquellos estudiantes de Educación Básica que más se destacaron, condicionadas a la disponibilidad de recursos por la agencia de fomento. Los estudiantes premiados en las diversas categorías fueron invitados a participar del Clube de Ciências Ciência Ativa do Laboratório de Divulgação Química do Sudoeste da Bahía (LADIO), en 2018. Las becas de Iniciación Científica fueron concedidas a cinco de estos estudiantes por criterios establecidos por la coordinación del club.

\section{Conclusiones}

Vila da Ciência representa la lucha desigual de la comunidad académico-científica en contra de la insensibilidad de un Estado que no valoriza el conocimiento y que, por lo tanto, no lo pone en el rol de sus prioridades, haya vista los cortes presupuestarios, así como la edificación de una política efectiva y consistente en el ámbito de la ciencia, lo que dificulta sacar al país de situaciones de vulnerabilidad social, económica, cultural y política.

Los integrantes del Proyecto Vila da Ciência defienden el acceso democrático al conocimiento como imprescindible en cualquier sociedad que vislumbre desarrollar las posibilidades de convivencia cada vez más humana y armónica entre todos y todas que en ella habitan. Los conciben como condición sine qua non al crecimiento social e individual, así como un aporte igualmente imprescindible para la disminución o misma eliminación de las exclusiones social, cultural, económica y política. 
A partir de ese ejemplo, cuando pensamos en los papeles que deben desarrollar los Estados, en todas las dimensiones de sus obligaciones, no podemos concebir la poca importancia dirigida a una política científica que ponga entre sus prioridades no solamente la construcción de conocimientos científicos y tecnológicos, sino también la apropiación de éstos por la sociedad en general. Sin, embargo, como vimos, América Latina insiste en desconsiderar la importancia de la ciencia, por diversas causas, lo que pone la región en situación de desventaja respecto a otras áreas del Globo, sobre todo cuanto a cuestiones que involucran directa o indirectamente desarrollo y calidad de vida.

En que pese la falta de comprometimiento del Estado brasileño con la promoción de una cultura científica, aún así, algunos esfuerzos son realizados por la comunidad académica, como el Proyecto de extensión Vila da Ciência, expuesto en este trabajo, lo cual demuestra no solamente el compromiso de profesionales de este sector, tanto en la construcción como también en la democratización del conocimiento científico, sobre todo involucrando a la sociedad, a través de la del intento de integración entre la Universidad y la comunidad.

El referido proyecto significa ayudar a mantener viva la posibilidad de cambio de rumbo, a través de la sensibilización de la sociedad cuanto al valor del conocimiento, de la universidad, así como la necesidad de percibir a esta institución en cuanto uno de los pilares imprescindibles al proceso de desarrollo humano y social. Por lo tanto, al involucrar personas en el proceso de construcción y divulgación de conocimiento el Vila da Ciência pasa a ser, además de un proyecto de extensión universitaria, una estrategia necesaria a la construcción de una cultura científica en Brasil. 


\section{Referencias bibliográficas}

ANGELO, Cláudio (2017): "Brazil's scientists plead to save funds: If budget levels do not increase soon, research institutions could start shutting down next year." Nature, 550, pp.166-167

BALBOLTíN, Rodrigo; ROESCHMANN, Juan Andrés \& ZAHLER, Andrés (2018). "Ciencia, Tecnología e Innovación en Chile: un análisis presupuestario": http://www.dipres.gob.cl/598/articles-17108o_doc_pdf.pdf. [fecha de consulta: 1 de octubre de 2018]

BRASIL (2015). "Percepção pública da ciência e tecnologia. Ciência e tecnologia no olhar dos brasileiros". Brasilia, Centro de Gestão em Estudos Estratégicos (CGEE), pp. 23: https://www.cgee.org.br/documents/10182/734063/percepcao_web.pdf. [fecha de consulta: 3 de octubre de 2018]

BUENO, Wilson da Costa (2014). "A Divulgação da Produção Científica no Brasil: A Visibilidade da Pesquisa nos Portais das Universidades Brasileiras". Revista Ação Midiática, n7:

https://revistas.ufpr.br/acaomidiatica/article/viewFile/36340/22901. [fecha de consulta: de octubre de 2018]

CASTELFRANCHI, Yurij; VILELA, Elaine Meire; BARRETO DE LIMA, Luciana; de CASTRO MOREIRA, Ildeu \& MASSARANI, Luisa (2013). "As opiniões dos brasileiros sobre ciência e tecnologia: o 'paradoxo' da relação entre informação e atitudes" Hist. cienc. saudeManguinhos [online]. v20, suppl.1. pp. 1163-1183: http://dx.doi.org/10.1590/S010459702013000400005. [fecha de consulta: 4 de octubre de 2018]

CASTRO, Guillermo Williamson \& FUENTES, Néstor Contreras (2018). "Universidad en el siglo XXI: ¿extensión, responsabilidad social universitaria, vínculo con el medio o conocimiento para el desarrollo de los territorios?" Espaço Pedagógico, v25, n3: http://http://seer.upf.br/index.php/rep/article/view/8566/114114234. [fecha de consulta: 5 de octubre de 2018]

CEBRELLI, Alejandra \& ARANCIBIA, Víctor (2017). "Los paisajes de la crisis Los desafíos del campo científico en la Argentina neoliberal". Cuadernos de ciencias sociales, v6, n8, pp. 49: http://revistas.unne.edu.ar/index.php/dpd/article/view/2369. [fecha de consulta: 5 de octubre de 2018]

GIRALDEZ, Lisandro, CASTELLUCIO, Ana Carolina, \& ALMEIDA, Verbena Córdula (2014). "Da pesquisa para a sociedade: reflexões sobre comunicação científica e tecnológica". Ilhéus, Editus Editora da UESC, pp. 28

LIMA, Marcelo de Oliveira \& VIANA, Giselle Rachid (2017). "Divulgação científica: responsabilidade e importancia". Revista Pan-Amaz Saude, v8, n4: http://scielo.iec.gov.br/pdf/rpas/v8n4/2176-6223-rpas-8-04-00007.pdf. [fecha de consulta: 5 de octubre de 2018]

PERUTZ, Max F. (1990). "Es necesaria la ciencia?" Madrid, Espasa Universidad, pp. 15 
PEREIRA, Ismael Soares \& KOSHIYAMA, Débora (2017). "Democratização do acesso à informação científica pela Biblioteca do Instituto do Cérebro UFRN". Revista Brasileira de Biblioteconomia e Documentação:

https://febab.emnuvens.com.br/rbbd/article/view/80g/859. [fecha de consulta: 5 de octubre de 2018]

PETHERICK, Ana (2017) "Austerity bites deeply: Institutions in Argentina and Brazil are struggling to maintain their funding and talent" Nature, 548, pp. 250-251.

RIBEIRO, Alcione. (2018, octubre). Entrevista. Alcione Ribeiro. Entrevista concedida a Verbena Córdula Almeida. A divulgação científica e o Projeto Vila da ciência (via correo electrónico).

SANABRIA-RANGEL, Pedro Emilio; MORALES-RUBIANO, María Eugenia \& ORTIZRIAGA, Carolina (2015). I"nteracción universidad y entorno: marco para el emprendimiento". Educación y educadores, v18, n1:

http://www.redalyc.org/pdf/834/83439194007.pdf. [fecha de consulta: 10 de octubre de 2018]

SOBRINHO, José Dias (2014). "Universidade e novos modos de produção, circulação e aplicação do conhecimento". Avaliação, v19, n3, pp. 643-662: http://submission.scielo.br/index.php/aval/article/view/141223/8932. [fecha de consulta: 12 de octubre de 2018]

TURCHI, Lenita Maria \& MORAIS, José Mauro (2017). "Políticas de apoio à inovação tecnológica no Brasil: avanços recentes, limitações e propostas de ações". Brasília, Ipea, 2017

UNGER, Maximilian \& POLT, Wolfgang. (2017). "The Knowledge Triangle between Research, Education and Innovation - A Conceptual Discussion". Foresight and STI Governance, $n$ 11, pp. 10-26

UNZUÉ, Martín \& ROVELLI, Laura Inés (2017). "Cambios, tendencias y desafíos de las políticas científicas recientes en las universidades nacionales de Argentina". Nueva Época, v42, pp. 242-261: http://www.scielo.org.mx/pdf/tla/v11n42/1870-6916-tla-11-4200242.pdf. [fecha de consulta: 13 de octubre de 2018]

VOGT, Geraldi; CECATTO, Ana Júlia \& CUNHA, Márcia Borin (2018). "A fotografia científica e as atividades experimentais: livros didáticos de química". ACTIO http://oaji.net/articles/2017/6441-1530402053.pdf. [fecha de consulta: 13 de octubre de 2018]. 